\title{
Achieving Minimal Residual Disease Negative Status in CLL - a Good Point to Start Thinking about Life without Treatment: How to Check MRD Status?
}

\section{Anna $\mathbf{G}$ and Agata AF* $^{*}$}

Department of Cancer Genetics with Cytogenetic Laboratory, Medical University of Lublin, Lublin, Poland

\begin{abstract}
Patients diagnosed with chronic lymphocytic leukemia (CLL) do not necessarily have to undergo intensive treatment. The implementation of the therapy depends on patient condition and the form of CLL which is determined using predictive and prognostic factors. Both indolent and aggressive forms of CLL should be monitored. To improve survival and the quality of life it is very important to know how the organism responds to the treatment. For this purpose, the estimation of the minimal residual disease (MRD) - the pool of persistent leukemia cells after or at the time of treatment was introduced. These cells resistant to treatment can lead to recurrence of the disease. There are several methods for MRD assessment, however in every case it is necessary to know the initial status of the tested alterations (chromosome aberrations, surface protein expression, etc.). It allows for assessment whether the treatment eliminated the leukemic cells. The most common method for MRD evaluation in CLL is flow cytometry. Intensive development of molecular biology techniques can contribute to implementation of other extremely sensitive and specific methods. This review presents the most recent state of the art concerning minimal residual disease in CLL, including methods that are used for MRD assessment.
\end{abstract}

Keywords: Chronic lymphocytic leukemia; Minimal residual disease; MRD assessment methods

\section{Introduction}

Chronic lymphocytic leukemia is a lymph proliferative disorder with features of cells accumulation. Congeries of small, morphologically mature but nonfunctional B cells are characteristic for this disorder. The accumulation of leukemic lymphocytes in peripheral blood, bone marrow, liver, spleen and lymph nodes is observed. CLL mainly affects people over 60 years of age, however, nowadays increasing prevalence of the disease in younger persons, even before 50 years of age, is observed [1,2]. CLL is a heterogeneous disease; in its course two principal forms are distinguished: indolent and aggressive. The former form is characterized by long survival, low risk of progression and decease. The treatment is frequently not required. In such cases, the strategy "watch and wait" is applied. It means the control of the clinical condition of the patient and taking into consideration the possibility of evolution to aggressive form of CLL [3,4]. Second principal type of CLL is associated with high risk of disease progression and short overall survival. Generally in patients diagnosed with that kind of CLL rapid implementation of therapy is required [3]. In CLL patients a variety of therapeutic strategies can be considered including monotherapy or combination therapy. The therapeutic options include use of purine analogs (fludarabine, pentostatin, cladribine), alkylating agents (chlorambucil, cyclophosphamide, bendamustine), corticosteroids (prednisone, dexamethasone) monoclonal antibodies (alemtuzumab, rituximab, ofatumumab) or blood stem cell transplantation [5-7]. Prior to treatment it should be determined whether the clinical condition of the patient will allow the implementation of a specific therapeutic strategy. Unfortunately, the selection and administration of specific therapy does not guarantee a complete cure. Some patients can achieve a state of complete remission (CR), the others achieve only partial response (PR). In many cases no response to treatment is observed and CLL progresses $[8,9]$. With the decision of implementation of particular therapy, the prognostic and predictive factors are helpful, such as: Rai disease staging, the expression of kinase ZAP-70 (zetachain-associated protein kinase 70 ), the expression of cell surface molecules CD38, mutational status of immunoglobulin heavy chain variable region $(\mathrm{IgVH})$, chromosomal aberrations, mutations within genes: TP53, NOTCH1, ATM, SF3B1 [10]. In the future also expression profiling of microRNA molecules (intracellular or circulating) may possibly complement the group of prognostic factors [11]. Minimal residual disease (MRD) is defined as a pool of cancer cells that survived the therapy. One of therapeutic strategies in leukemia is bone marrow transplantation. CML was one of the first leukemia's, which was successfully treated by using allogeneic bone marrow transplantation [12]. Then it turned out that the RT-PCR (reverse transcription polymerase Chain reaction) molecular technique provides an excellent tool for predicting disease relapse after such treatment [13]. CML is characterized by balanced translocation between chromosomes 9 and 22, which results in specific Philadelphia ( $\mathrm{Ph}$ ') chromosome formation. Based on the identification of this chromosome or his molecular equivalent - BCR/ABL1 fusion gene, CML is diagnosed. The number of Ph'(+) cells or the level of BCR/ABL1 transcript (assessed by means of RT-PCR) after treatment stands for minimal residual disease, and its evaluation enables to assess the cytogenetic or molecular response to the therapy [14]. Minimal residual disease (MRD) estimation in the treatment and monitoring of CLL is of great importance. This is due to the fact that a small percentage of leukemia cells can remain alive despite intensive treatment, and they may be the cause of CLL relapse. Nowadays the main aim of first-line therapy is to achieve a state of complete clinical remission, which is assumed as a state negative for

*Corresponding author: Agata AF, Department of Cancer Genetics with Cytogenetic Laboratory, Medical University of Lublin, 11 Radziwillowska Str, 20 080 Lublin, Poland, Tel: (0048) 8144861 02; E-mail: aafilip@hotmail.com

Received July 12, 2017; Accepted July 29, 2017; Published August 01, 2017

Citation: Anna G, Agata AF (2017) Achieving Minimal Residual Disease Negative Status in CLL - a Good Point to Start Thinking about Life without Treatment: How to Check MRD Status? J Blood Lymph 7: 170. doi: 10.4172/2165-7831.1000170

Copyright: ( 2017 Anna G, et al. This is an open-access article distributed under the terms of the Creative Commons Attribution License, which permits unrestricted use, distribution, and reproduction in any medium, provided the original author and source are credited. 
minimal residual disease [15]. Early achieving the negative status of MRD is advantageous because of the opportunity to discontinue burdensome and exhaustive treatment.

\section{Minimal residual disease in CLL}

It is known that the elimination of CLL clinical symptoms, which is result of the therapy application, does not necessarily imply the complete disease eradication. In many cases, a pool of leukemia cells that may contribute to the relapse of the disease could remain in the blood or bone marrow even after treatment. Therefore it is important to determine the presence and the number of persistent leukemia cells in individual patients, treated according to the specific therapeutic regimens, because the evaluation of MRD can change the therapeutic strategy and may improve the clinical response [16].

The purpose of therapy is to achieve complete remission (CR) which means regression of disease symptoms and achieving of blood counts acceptable for a given age range. No infiltration of leukemic cells in the bone marrow and the presence of less than $30 \%$ of lymphocytes in bone marrow sample are also important. Patients who achieved CR present longer survival in comparison to patients which are characterized by poor response to treatment [17]. Unfortunately, even in patients with clinical CR, persistent CLL cells may lead to a deterioration of the condition and disease relapse. For this reason, it is important to implement of a therapy, which could eliminate the MRD [17]. Currently, it is assumed that the patient is MRD negative, when less than one leukemic cell per 10000 white blood cells or less than 1 leukemic cell per 1000 lymphocytes is detected [17,18]. Number of detected cells depends on the method of detection and their sensitivity $[17,18]$. The observed eradication of leukemia cells using commercially available methods can be crucial for the decision to complete the therapy. It has been proved that the assessment of MRD is helpful in estimating overall survival (OS), treatment-free survival (TFS) and progression-free survival (PFS), in the context of the treatment $[5,19]$.

\section{Impact of CLL biology and treatment on minimal residual disease}

Current therapeutic approaches take into account cytogenetic and molecular characteristics of CLL patients. These are extremely important in choosing the most appropriate time and type of treatment. Using FISH (fluorescence in situ hybridization) chromosome aberrations such as deletions of 13q14.3, 11q22-23, 17p.13 or trisomies of chromosome 12 in CLL are estimated [20-23]. Among the group of molecular prognostic and predictive factors, the most important are mutations of genes: TP53, ATM, and more recently discovered mutations in NOTCH1 and SF3B [24-28]. Both deletion and mutation of TP53 gene (locus 17p13) are of great importance for the decision about implementation of therapy. These lesions are unfavorable prognostic factors, indicating the aggressive course of the disease. TP53 deleted/ mutated CLL cases require rapid treatment implementation [20,24]. Despite such approach, some CLL cases are treatment resistant, which worsens the prognosis. Good example is the common therapeutic strategy FCR (fludarabine-cyclophosphamide-rituximab), which does not seem to be suitable for patients diagnosed with mutations/deletions of TP53. In such patients treated with the FCR regimen the proportion of achieving complete remission is about $5 \%$, as compared with $50 \%$ in CLL patients without TP53 lesions [6]. Therefore, molecular and cytogenetic characterization has a great impact on the selection of first line therapy. Previous reports indicated that the negative MDR was associated with a significantly longer duration of progression-free survival and longer overall survival $[5,15,19]$. In addition, it turned out that MRD status was as important as the well-known prognostic factors such as chromosomal aberrations, expression of ZAP-70, mutations in NOTCH1 and SF3B1 [5]. CLL patients achieving MRD negative status and complete response after one of the first line therapies (chlorambucil, fludarabine-rituximab-cyclophosphamide, fludarabine-cyclophosphamide/fludarabine-cyclophosphamidemitoxantrone; cladribine/fludarabine, cyclophosphamide-vincristineAdriamycin-prednisone) are characterized by median treatment-free survival of 76 months [5]. Patients who are minimal residual disease positive $(\mathrm{MRD}+)$ with complete response, partial and no response to one of the first line therapies are Characterized by median treatmentfree survival of 40, 11 and 11 months, respectively [5]. It is known that implementation of the first-line treatment strategy is most favorable for eradication of CLL. Achieving of MRD negative status can be one of the most useful factors, which might indicate a longer survival time. The difference in overall survival is apparent between groups of treated patients depending on MRD status. The OS is significantly prolonged for patients with a complete response to therapy in comparison to patients with partial response to treatment [5]. Achieving the negative MRD status after front-line therapy is also associated with significantly prolonged treatment-free survival irrespective of the type of treatment and other adverse prognostic markers (i.e. aberrations of chromosomes 17 and 11, unmutated IGVH, mutations of TP53, NOTCH1 and SF3B1, CD38 and ZAP70 over-expression) [5]. One of the common therapies in CLL is based on alkylating agents. Such treatment enables to control the course of the disease, however in most cases it does not allow to decrease the count of lymphocytes to the level, which would be sufficient to prolong the survival time. It is important because the higher is the number of persistent leukemia cells after treatment, the higher risk of relapse and reduced survival time is observed. For this reason treatment strategy is aimed at MRD negative status achievement in first-line therapy. This condition in most of cases indicates low risk of the disease relapse and is associated with long-lasting remission [29]. In patients at high risk of disease progression, with an unfavorable prognosis, exhibiting resistance to chemotherapy it is possible to use alemtuzumab [30]. It is a monoclonal antibody specifically binding to the CD52 antigen located on the surface of normal and leukemic B and $\mathrm{T}$ lymphocytes. This antibody can be used as a first-line therapy, contributing to the elimination of minimal residual disease [30] Application of alemtuzumab has the important advantage - it targets the lymphocytes present in blood, bone marrow or spleen, but does not affect hematopoietic stem cells. Thus, it enables eradication of leukemic cells from peripheral blood and bone marrow, and saves bone marrow being able to produce normal lymphocytes from the stem cells.

\section{Methods for MRD assessment in CLL}

The possibility to determine the number of leukemic cells remaining after treatment is helpful in monitoring the course of the disease or long-term complete remission. There are two principal methods for MDR assessment: flow cytometry (FC) and real-time PCR (qPCR, quantitative PCR). The former technique is based on differences in the expression of particular surface proteins between the leukemic and normal cells. It utilizes fluorescent labeled antibodies that specifically bind to the cell surface antigens. In this technique distinguishing of CLL cells from other blood cells, particularly normal B and T lymphocytes, is essential. Flow cytometry is utilized for immunophenotyping during CLL diagnostics, but it also seems to be effective in MRD assessment [31,32]. Uncomplicated approach for flow cytometry for this purpose may be targeting the surface marker CD19/CD5 and pre-analyzing the clonal co-expression of surface $\kappa / \lambda$ light chains. This semi-quantitative method is not perfect because of variable sensitivity and specificity. 
However, it might be useful in some cases, when using highly sensitive methods for MRD estimation is not necessary, for example in the cases of expected high counts of persistent leukemia cells [33].

\section{MRD evaluation using 4-color labeled antibodies for flow cytometry}

Rawstron et al. suggested methodology of 4-color antibodies labeling for flow cytometry (FC) in order to estimate MRD in CLL [34]. This method was validated in the multicenter system with randomized clinical trials. Protocol for the four-marking assumes using the antibodies: anti-CD14/3/45/19, anti-CD20/38/5/19, antiCD22/81/5/19, anti-CD79b/43/5/19 [34]. Currently, this method is recommended and widely used for the evaluation of minimal residual disease in CLL. It has a number of advantages and disadvantages. Due to the latter, there are attempts to develop and implement more sensitive and specific methods for assessing MRD in CLL for everyday laboratory practice. One of such methods, ASO-IGH RQ-PCR is presented.

\section{MRD evaluation using 8-color labeling antibodies for flow cytometry}

Stehlíková et al. and Raponi et al. suggested an 8-color labeling antibodies methodology application for the FC in order to enhance detection of MRD as well as to reduce the difficulties associated with the preparation of samples for laboratory analysis [17,35]. Stehlíková has used the regime with two tubes which seems to be easier and less time-consuming then 4-color labeling method utilizing 4 tubes. In 8-color labeling technique the first tube contained the antibodies anti-CD3/5/19/22/43/45/79b/81, and the second contained antiCD5/14/19/20/38/43/45/79b [17]. The mixture of labeled antibodies in the first tube was used for a proper assessment of MRD, while the mixture of the second tube constituted a confirming control. It turned out that both 4 and 8-color labeling techniques used in detection of MRD are comparable ( $100 \%$ concordance in detecting MRD positivity) [17]. Stehlíková concluded that two FC techniques are similar; however sample preparing could be easier in 8-color method. On the other side in the latter technique the usage of a special kind of cytometers is necessary, which are not available in every laboratory.

\section{MRD evaluation using ASO IGH RQ-PCR technique}

The validity of PCR technique in MRD monitoring has already been established for acute lymphoblastic leukemia (ALL), acute and chronic myelogenous leukemia (AML, CML) and Hodgkin's lymphoma [36]. The use of molecular methods in MRD assessment is, however, still not applied in everyday laboratory practice. Stehlíková et al. have compared flow cytometry and ASO IGH RQ-PCR method (real-time quantitative allele-specific oligonucleotide IGH polymerase chain reaction) for MRD assessment [17]. It has been found that ASO IGH RQ-PCR was more sensitive and enabled detection of 1 CLL cell per 1000000 leukocytes [17]. For that method specific primers and molecular TaqMan probes have been designed, which contained LNA (locked nucleic acids) bases [37]. The usage of modified bases makes the probes stable. It is because of methylene bridges content in molecules. This improves the binding force of the probe to the PCR products (amplicons) and enables more precise detection of fluorescence signal from the probe by the real-time system. The probes are designed for IGVH gene fragments (heavy chain variable region of IGH gene) for leukemic cell clones, individually for each patient. This method is timeconsuming, complicated and expensive, and for these reasons it is not used in everyday clinical practice. Another difficulty is the necessity of designing PCR primers for each patient. Raponi et al. have taken into consideration the direct sequencing of DNA before designing primers for ASO IGH RQ-PCR reaction [35]. This approach is expensive, and the validation of the method for the implementation to the daily clinical practice is extremely difficult.

\section{MRD evaluation using high throughput sequencing (HTS)}

High throughput sequencing (HTS) to assess MRD has been described by Logan et al. $[38,39]$. They suggested that this method enables the elimination the difficulties associated with the individual primer design in ASO IGH RQ-PCR technique. HTS method utilizes the degenerate primers for the amplification of IGH genes in a mixture of polyclonal lymphoid cells $[38,39]$. Degenerate primers contain chemically modified bases, which can bind to different types of deoxy nucleotides in the examined DNA. It is beneficial because of IGH gene rearrangements resulting in such changes in the nucleotide sequence, that the final sequence of a tested DNA is not exactly known. This phenomenon brings about the necessity of designing primers and molecular probes specific for the clones of leukemic cells for individual patient in ASO IGH RQ-PCR method. The HTS technology eliminates this problem by using degenerated primers. Degenerated primers are a mix of oligonucleotides of similar but not identical sequence [40]. Annealing temperature for PCR (polymerase chain reaction) with this kind of primers should be slightly lower than for conventional reaction. If annealing temperature is standard, only primers witch $100 \%$ complementarity will anneal to the examined DNA. It is undesirable condition in usage of HTS method. HTS technique allows for increasing the number of DNA regions amplified, which are similar but not identical in sequence. This example presents the possibility of obtaining six different, but similar products in one reaction. This approach is essential for HTS method. Logan et al. used three sets of degenerate primers (multiplex reaction) for regions $\mathrm{V}$ (Variable) and one primer for IGH region J (Joining) to create a genomic library the step, which is necessary in the HTS procedure [38]. The presence of three sets of primers ensures successful amplification of IGH gene regions that underwent somatic hyper mutation. Next steps of the HTS method include linking of the obtained amplicons to the adaptors, clustering of clones, and amplification followed by sequencing. Logan et al. have used high throughput sequencing methods for MRD assessment in patients with CLL treated with allogeneic stem cells transplantation [38]. They concluded that the HTS method has high sensitivity and specificity and may be useful in clinical practice for evaluation of MRD and CLL relapse risk after stem cell transplantation [38]. Despite all advantages it seems that it is not possible yet to use this procedure in every diagnostic and therapeutic unit. It requires modern instruments, specific and expensive reagents, which are unavailable in many laboratories.

\section{Conclusion}

Recent studies gained enough evidence to include the evaluation of MRD in the clinical guidelines of CLL management. MRD negative status in CLL is considered as good prognostic factor. The intensive development of molecular biology techniques can contribute to the usage of methods such as ASO IGH RQ PCR or high throughput sequencing (HTS) for MRD assessment. The implementation of that kind of methods requires multicenter studies, standardization and validation. However, their benefits are transparent and every possible effort should be made to utilize them for evaluation of MRD in everyday clinical practice. This may facilitate the work of clinicians, and - before all - improve the survival and quality of life of CLL patients. 
Citation: Anna G, Agata AF (2017) Achieving Minimal Residual Disease Negative Status in CLL - a Good Point to Start Thinking about Life without Treatment: How to Check MRD Status? J Blood Lymph 7: 170. doi: 10.4172/2165-7831.1000170

\section{References}

1. Redaelli A, Laskin BL, Stephens JM, Botteman MF, Pashos CL, et al. (2004) The clinical and epidemiological burden of chronic lymphocytic leukaemia. Eur J Cancer Care (Engl) 13: 279-287.

2. Conzen SD, Gottlob K, Kandel ES, Khanduri P, Wagner AJ, et al. (2000) Induction of cell cycle progression and acceleration of apoptosis are two separable functions of c-Myc: transrepression correlates with acceleration of apoptosis. Mol Cell Biol 20: 6008-6018.

3. Gribben JG (2010) How I treat CLL up front. Blood 115: 187-197.

4. Stilgenbauer S (2015) Prognostic markers and standard management of chronic lymphocytic leukemia. Hematology Am Soc Hematol Educ Program 2015: 368-377.

5. Santacruz R, Villamor N, Aymerich M, Martínez-Trillos A, López C, et al. (2014) The prognostic impact of minimal residual disease in patients with chronic lymphocytic leukemia requiring firstline therapy. Haematologica 99: 873-880.

6. Van Den NE, Letestu R, Aurran-Schleinitz T, Ysebaert L, Feugier P, et al (2012) Post-remission intervention with alemtuzumab or rituximab to eradicate minimal residual disease in chronic lymphocytic leukemia: where do we stand? Leuk Lymphoma 53: 362-370.

7. Bauer K, Rancea M, Roloff V, Elter T, Hallek M, et al. (2012) Rituximab, of atumumab and other monoclonal anti-CD20 antibodies for chronic lymphocytic leukaemia. Cochrane Database Syst Rev. 2012; 11: CD008079.

8. Hallek M, Cheson BD, Catovsky D, Caligaris-Cappio F, Dighiero G, et al (2008) International Workshop on Chronic Lymphocytic Leukemia. Guidelines for the diagnosis and treatment of chronic lymphocytic leukemia: a report from the International Workshop on Chronic Lymphocytic Leukemia updating the National Cancer Institute-Working Group 1996 guidelines. Blood 111: 54465456 .

9. Döhner H, Fischer K, Bentz M, Hansen K, Benner A, et al. (1995) p53 gene deletion predicts for poor survival and non-response to therapy with purine analogs in chronic B-cell leukemias. Blood 85: 1580-1589.

10. Filip AA (2013) New boys in town: prognostic role of SF3B1, NOTCH1 and other cryptic alterations in chronic lymphocytic leukemia and how it works. Leuk Lymphoma 54: 1876-1881.

11. Stamatopoulos B, Van DM, Crompot E, Dessars B, Housni HE, et al (2015) Opposite Prognostic Significance of Cellular and Serum Circulating MicroRNA-150 in Patients with Chronic Lymphocytic Leukemia. Mol Med 21:123-133.

12. Thomas ED, Clift RA, Fefer A, Appelbaum FR, Beatty $P$, et al. (1986) Marrow transplantation for the treatment of chronic myelogenous leukemia. Ann Intern Med 104: 155-163.

13. Hughes TP, Morgan GJ, Martiat P, Goldman JM (1991) Detection of residual leukemia after bone marrow transplant for chronic myeloid leukemia: role of polymerase chain reaction in predicting relapse. Blood 77: 874-878.

14. Bagg A (2002) Chronic myeloid leukemia: a minimalistic view of post therapeutic monitoring. J Mol Diagn 4: 1-10.

15. Strati P, Keating MJ, O'Brien SM, Burger J, Ferrajoli A, et al. (2014) Eradication of bone marrowminimal residual disease may prompt early treatment discontinuation in CLL. Blood 123: 3727-3732.

16. Ghia P (2012) A look into the future: can minimal residual disease guide therapy and predict prognosis in chronic lymphocytic leukemia? Hematology Am Soc Hematol Educ Program 2012: 97-104.

17. Stehlíková O, Chovancová J, Tichý B, Krejčí M, Brychtová Y, et al. (2014) Detecting minimal residual disease in patients with chronic lymphocytic leukemia using 8-color flow cytometry protocol in routine hematological practice. Int J Lab Hematol 36: 165-171.

18. Varghese AM, Rawstron AC, Hillmen $P$ (2010) Eradicating minimal residual disease in chronic lymphocytic leukemia: should this be the goal of treatment? Curr Hematol Malig Rep 5: 35-44.

19. Böttcher S, Ritgen M, Fischer K, Stilgenbauer S, Busch RM, et al. (2012) Minima residual disease quantification is an independent predictor of progression-free and overall survival in chronic lymphocytic leukemia: a multivariate analysis from the randomized GCLLSG CLL8 trial. J Clin Oncol 30: 980-988.

20. Zenz T, Häbe S, Denzel T, Mohr J, Winkler D, et al. (2009) Detailed analysis of p53 pathway defects in fludarabine-refractory chronic lymphocytic leukemia
(CLL):dissecting the contribution of 17p deletion, TP53 mutation, p53-p21 dysfunction, and miR34a in a prospective clinical trial. Blood 114: 2589-2597.

21. Bacher U, Kern W, Schoch C, Hiddemann W, Haferlach T, et al. (2004) Discrimination of chronic lymphocytic leukemia (CLL) and CLL/PL by cytomorphology can clearly be correlated to specific genetic markers as investigated by interphase fluorescencein situ hybridization (FISH). Ann Hematol 83: 349-355.

22. Kiefer Y, Schulte C, Tiemann M, Bullerdiek J (2012) Chronic lymphocytic leukemia associated chromosomal abnormalities and miRNA deregulation. The Application of Clinical 491 Genetics 5: 21-28.

23. Döhner H, Stilgenbauer S, Benner A, Leupolt E, Kröber A, et al. (2000) Genomic aberrations and survival in chronic lymphocytic leukemia. N Engl J Med 343: 1910-1916.

24. Zenz T, Eichhorst B, Busch R, Denzel T, Häbe S, et al. (2010) TP53 12 mutation and survival in chronic lymphocytic leukemia. J Clin Oncol 28: 4473-4479.

25. Guarini A, Marinelli M, Tavolaro S, Bellacchio E, Magliozzi M, et al. (2012) ATM gene alterations in chronic lymphocytic leukemia patients induce a distinct gene expression profile and predict disease progression. Haematologica 97: 47-55.

26. Willander K, Dutta RK, Ungerbäck J, Gunnarsson R, Juliusson G, et al. (2013) $\mathrm{NOTCH} 1$ mutations influence survival in chronic lymphocytic leukemia patients. BMC Cancer 13: 274.

27. Quesada V, Conde L, Villamor N, Ordóñez GR, Jares P, et al. (2011) Exome sequencing identifies recurrent mutations of the splicing factor SF3B1 gene in chronic lymphocytic leukemia. Nat Genet 44: 47-52.

28. Ramsay AJ, Quesada V, Foronda M, Conde L, Martínez-Trillos A, et al. (2013) POT1 mutations cause telomere dysfunction in chroniclymphocytic leukemia. Nat Genet 45: 526-530.

29. Montillo M, Schinkoethe T, Elter T (2005) Eradication of minimal residua disease with alemtuzumab in B-cell chronic lymphocytic leukemia (B-CLL) patients: the need for a standard method of detection and the potential impact of bone marrow clearance on disease outcome. Cancer Invest 23: 488-496.

30. Schweighofer CD, Wendtner CM (2010) First-line treatment of chronic lymphocytic leukemia: role of alemtuzumab. Onco Targets Ther 3: 53-67.

31. Jilani I, O'Brien S, Manshuri T, Thomas DA, Thomazy VA, et al. (2003) Transient down-modulation of CD20 by rituximab in patients with chronic lymphocytic leukemia. Blood 102: 3514-3520.

32. Rawstron AC, de Tute R, Jack AS, Hillmen P (2006) Flow cytometric protein expression profiling as a systematic approach for developing disease-specific assays: identification 13 of a chronic lymphocytic leukaemia-specific assay for use in rituximab-containing regimens. Leukemia 20: 2102-2110.

33. Tam CS, O'Brien S, Wierda W, Kantarjian H, Wen S, et al. (2008) Long-term results of the fludarabine, cyclophosphamide, and rituximab regimen as initial therapy of chronic lymphocytic leukemia. Blood 15:112: 975-980.

34. Rawstron AC, Villamor N, Ritgen M, Böttcher S, Ghia P, et al. (2007) International standardized approach for flow cytometric residual disease monitoring in chronic lymphocytic leukaemia. Leukemia 21: 956-964.

35. Raponi S, Della SI, De Propris MS, Del GI, Mauro FR, et al. (2014) Minima residual disease monitoring in chronic lymphocytic leukaemia patients. A comparative analysis of flow cytometry and ASO IgH RQ-PCR. Br J Haematol 166: $360-368$

36. Liang R, Chan D, Kwong YL, Chan V (1997) Molecular detection of minima residual disease for patients with leukaemia and lymphoma. HKMJ 3: 195-200.

37. Uhrmacher S, Erdfelder F, Kreuzer KA (2010) Flow cytometry and polymerase chain reactionbased analyses of minimal residual disease in chronic lymphocytic leukemia. Adv Hematol 2010: 272517.

38. Logan AC, Zhang B, Narasimhan B, Carlton V, Zheng J, et al. (2013) Minima residual disease quantification using consensus primers and high-throughput IGH sequencing predicts post-transplant relapse in chronic lymphocytic leukemia. Leukemia. 2013; 27: 1659-1665.

39. Logan AC, Gao H, Wang C, Sahaf B, Jones CD, et al. (2011) High throughput VDJ sequencing for quantification of minimal residual disease in chronic lymphocytic leukemia and immune reconstitution assessment. Proc Natl Acad Sci USA 108: 21194-21199.

40. Linhart C, Shamir R (2005) The degenerate primer design problem: theory and applications. J Comput Biol 12: 431-456. 